\title{
HIV self-testing in Ottawa, Canada used by persons at risk for HIV: The GetaKit study
}

\author{
Patrick O’Byrne ${ }^{1 *}$, Alexandra Musten², Amanda Vandyk', Nikki Ho', Lauren Orser', \\ Marlene Haines ${ }^{1}$, Vickie Paulin ${ }^{3}$
}

\begin{abstract}
Background: The Public Health Agency of Canada estimates that about $87 \%$ of persons living with human immunodeficiency virus (HIV) in Canada have been diagnosed, which is well below the Joint United Nations Programme on HIV/AIDS target to have 95\% of HIV-positive persons diagnosed. Research has shown that HIV self-testing may help increase such diagnoses, especially among the populations who are most affected by HIV. The objective of the study was to determine the uptake and diagnosis outcomes associated with free HIV self-testing.

Methods: We developed the first online mailout free HIV self-testing program in Canada and implemented it in Ottawa. This project ran through the website, www. GetaKit.ca. We intended to recruit 150-400 participants over a 6-12-month period, estimating that this number would yield between $0-1$ positive test results (expected positivity rate of $0.08 \%$ ).
\end{abstract}

Results: Between July 20, 2020 and April 1, 2021, 1,268 people accessed the GetaKit website and verified their eligibility. In total, 600 persons were eligible and 405 ordered an HIV kit. Of those who ordered a kit, 399 completed a baseline survey. Overall, $71 \%$ of these participants were members of HIV priority groups. For test results, 228 persons reported test results, with one being positive, for a positivity rate of $0.24 \%$ overall and $0.44 \%$ of reported results. These rates exceed that normally observed in Ottawa.

Conclusion: Self-testing of HIV can be effectively delivered through a website. Such an intervention will also be used by persons with undiagnosed infections and appears to do so at a rate higher than that observed by other means of testing. Self-testing of HIV may therefore help Canada achieve the United Nations 95-95-95 targets.
This work is licensed under a Creative Commons Attribution 4.0 International License.

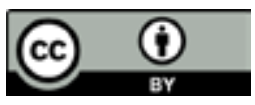

Affiliations

${ }^{1}$ School of Nursing, University of Ottawa, Ottawa, ON

${ }^{2}$ Ontario HIV Treatment Network, Toronto, ON

${ }^{3}$ Ottawa Public Health, Ottawa, ON

\section{*Correspondence:}

patrick.obyrne@uottawa.ca

Suggested citation: O'Byrne P, Musten A, Vandyk A, Ho N, Orser L, Haines M, Paulin V. HIV self-testing in Ottawa, Canada used by persons at risk for HIV: The GetaKit study. Can Commun Dis Rep 2021;47(10):435-41. https://doi.org/10.14745/ccdr.v47i10a06

Keywords: HIV, self-test, Ottawa, Canada, priority group, GetaKit

\section{Introduction}

The Joint United Nations Programme on HIV/AIDS 95-95-95 targets aim to have $95 \%$ of persons living with human immunodeficiency virus (HIV) diagnosed, $95 \%$ of those diagnosed engaged in care and $95 \%$ of those in care achieving and maintaining a suppressed HIV viral load by 2030 (1). However, the Public Health Agency of Canada (2) estimates that in 2018 only $87 \%$ of HIV-positive persons in Canada were diagnosed. Moreover, PHAC data highlight that in addition to approximately $13 \%$ of persons remaining undiagnosed, HIV continues to unequally affect the same priority groups: gay, bisexual and other men who have sex with men (gbMSM); persons who are transgender; individuals of African, Caribbean or Black ethnicities; members of Indigenous communities; and persons who use drugs $(3,4)$. One factor that likely contributes to this ongoing transmission and to why persons remain unaware of their HIV-positive status is persistent barriers to current methods of HIV testing, including at the individual level (fear of results, concerns about confidentiality, etc.), at the healthcare provider level (stigma, reluctance to test, etc.), and at the institutional/ policy level (criminalization of behaviour, limited resources, etc.) (5).

Because HIV self-testing, compared to peer and clinic-based testing, often corresponds to increased testing, diagnosis and reported user satisfaction among members of HIV priority groups (6-9), we studied the outcomes associated with free 
at-home HIV self-testing in Ottawa. To accomplish this, we launched GetaKit (www.GetaKit.ca), which was the first online mailout program in Canada through which individuals could order an INSTI ${ }^{\circledR}$ HIV self-test and have it delivered to their home or other designated pick-up location. While other studies have observed patients completing self-testing in controlled clinical settings, in implementing GetaKit, our goals were to: 1) evaluate HIV self-testing in real-world settings, 2) facilitate HIV testing, 3) identify persons with undiagnosed HIV infection and 4) link persons to care or prevention services depending on their HIV test result. While we have detailed GetaKit implementation elsewhere, herein we report findings from July 20, 2020 to April 1, 2021 and describe our participants, including details about the number who belonged to a priority group or who identified as women; we also report on the correlates of first-time testers and persons who reported their results.

\section{Methods}

\section{Design}

GetaKit is an open-cohort prospective observational study with three phases. Phase 1 piloted free at-home HIV self-tests in Ottawa. Because test positivity for HIV was $0.1 \%$ in Ontario and $0.08 \%$ in Ottawa (unpublished data-available upon request), a 6-12-month period was deemed sufficient to enroll 150-400 adults who could test up to three times each; we expected 0-1 positive result from this sample. Phase 2 involved self-test delivery in additional sites in Ontario. Phase 3 involved the addition of full sexually transmitted infection testing. This article reports on Phase 1.

\section{Data collection}

To be eligible, persons had to be HIV-negative or of unknown HIV-status, 18 years of age or older, live in or around Ottawa and have a cellular phone. Exclusion criteria included being on pre-exposure prophylaxis (PrEP), being in an HIV vaccine trial and having a diagnosed bleeding disorder.

For recruitment, we created GetaKit.ca and engaged in public awareness through posters in public places and healthcare centres and via social media. We worked with local acquired immunodeficiency syndrome service organizations to promote to priority groups.

Data collection occurred via GetaKit.ca. Stepwise, potential participants had to first complete an anonymous eligibility screening test, for which all questions were obligatory. Ineligible persons were referred to other resources for testing and support. Eligible persons could register, which involved providing a name, date of birth and cellular phone number (for two-factor authentication). Once registered, participants were asked to complete a survey, which collected information about country of birth, ethnicity, sex, gender, sexual orientation, sex and drug use practices and HIV testing history; "prefer not to answer" was an option in the survey. Once completed, participants could order an HIV self-test, which would be delivered in 1-3 business days. The self-test and shipping were free. We asked, but did not require, participants to report their HIV self-test results via GetaKit.ca.

The Ontario HIV Treatment Network funded GetaKit and the University of Ottawa Research Ethics Board approved the project (H-12-20-6450).

\section{Data analysis}

Data were extracted from Getakit.ca into an Excel file. Participant characteristics were reported for the total sample using frequencies and percentages. We stratified by gender, described the participants who identified as women using frequencies and percentages, and used bivariate $X^{2}$ to determine which characteristics differed significantly between groups. For outcomes of interest, we sought to understand which participants: 1) had previously completed HIV testing, 2) reported their HIV self-test results and 3) completed the HIV self-test appropriately (i.e. received a valid result). Each outcome was dichotomous, and independent variables (i.e. participant characteristics) were categorized to ensure adequate cell size. Relationships among independent variables and outcomes were first explored using bivariate binary logistic regression. If a significant relationship (in any direction) was identified at $p<0.1$, the variable was retained for multivariable analysis using hierarchical binary logistic regression. Each outcome was explored separately, and only variables significant at $p<0.05$ were included in the final models. Goodness of fit was assessed using the Hosmer-Lemeshow test. Cases with missing data were deleted listwise. SPSS v.26 was used for analysis.

\section{Results}

Phase 1 of our study lasted from July 20, 2020 to April 1, 2021. During this time, 1,268 persons submitted the eligibility screening test, averaging 160 accesses per month; $47.3 \%$ $(n=600)$ were eligible to register for a self-test. Notably, $59.1 \%$ $(n=395)$ of persons were ineligible after submitting incomplete data. Among the 273 persons with complete data, 14.3\% $(n=39)$ were ineligible for multiple reasons and the rest for single reasons. As summarized in Table 1, residing outside Ottawa was the most common reason for ineligibility, followed by pre-exposure prophylaxis use.

Of 600 eligible participants, $67.5 \%(n=405)$ completed a survey and ordered an HIV self-test. Six participants selected "prefer not to report" for all answers and were removed from the analysis. The remaining 399 participants were on average 32 years old, with $66 \%(n=264)$ reporting they were white, 68\% $(n=270)$ identifying as male, $57.4 \%(n=229)$ indicating they were gbMSM. As well, $57.1 \%(n=228)$ reported an income more than $\$ 40,000$ and $77 \%$ reported having College or University level education 


\section{Table 1: Reasons for participant ineligibility for HIV self-testing for Getakit.ca program}

\begin{tabular}{|l|r|r|r|r|}
\multicolumn{1}{|c|}{$\begin{array}{c}\text { Reason for } \\
\text { ineligibility }\end{array}$} & $\begin{array}{c}\text { Number of } \\
\text { individuals } \\
\text { (non- } \\
\text { exclusive) }\end{array}$ & $\begin{array}{c}\text { \% of } \\
\text { individuals } \\
\text { (non- } \\
\text { exclusive) }\end{array}$ & $\begin{array}{c}\text { Number of } \\
\text { individuals } \\
\text { (exclusive) }\end{array}$ & $\begin{array}{c}\text { \% of } \\
\text { individuals } \\
\text { (exclusive) }\end{array}$ \\
\hline $\begin{array}{l}\text { Live outside } \\
\text { Ottawa }\end{array}$ & 150 & 49 & 125 & 51 \\
\hline On PrEP & 85 & 28 & 67 & 27 \\
\hline No cell phone & 32 & 10 & 26 & 11 \\
\hline $\begin{array}{l}\text { Younger than } 18 \\
\text { years of age }\end{array}$ & 17 & 6 & 10 & 4 \\
\hline $\begin{array}{l}\text { HIV test result } \\
\text { (indeterminate } \\
\text { positive) }\end{array}$ & 10 & 3 & 7 & 3 \\
\hline Bleeding disorder & 9 & 3 & 7 & 1 \\
\hline $\begin{array}{l}\text { In an HIV vaccine } \\
\text { trial }\end{array}$ & 4 & 1 & 2 & 3 \\
\hline
\end{tabular}

Abbreviations: HIV, human immunodeficiency virus; PrEP, pre-exposure prophylaxis Non-exclusive denotes that this was one of many reasons why a participant was excluded exclusive denotes that this was the only reason why this person was deemed ineligible b Persons with indeterminate and positive results should undergo serology as follow-up

(ongoing or completed). In total, $70.9 \%(n=283)$ of participants had one or more characteristic of an HIV priority group, which rose to $76.4 \%(n=305)$ when all racialized persons were included in this analysis (Table 2).

Table 2: Characteristics of eligible participants for Getakit.ca program

\begin{tabular}{|c|c|c|c|}
\hline Characteristic & Description & $\mathbf{n}$ & $\%$ \\
\hline \multirow{2}{*}{$\begin{array}{l}\text { Member of a priority } \\
\text { population }(n=399)\end{array}$} & Yes & 283 & 71 \\
\hline & No & 116 & 29 \\
\hline \multirow{3}{*}{ Age (years) $(n=395)$} & 25 years or younger & 110 & 28 \\
\hline & 26-49 years old & 257 & 65 \\
\hline & 50 years and older & 28 & 7 \\
\hline \multirow{8}{*}{ Ethnicity ( $n=399$ ) } & Arab & 16 & 4 \\
\hline & Black & 23 & 6 \\
\hline & Indigenous & 16 & 4 \\
\hline & Latin & 13 & 3 \\
\hline & Mixed & 22 & 6 \\
\hline & South Asian & 13 & 3 \\
\hline & Southeast Asian & 25 & 6 \\
\hline & White & 264 & 66 \\
\hline \multirow{3}{*}{ Gender $(n=395)$} & $\begin{array}{l}\text { Men (includes transgender } \\
\text { men) }\end{array}$ & 270 & 68 \\
\hline & $\begin{array}{l}\text { Women (includes } \\
\text { transgender women) }\end{array}$ & 115 & 29 \\
\hline & Gender non-conforming & 10 & 3 \\
\hline \multirow{3}{*}{$\begin{array}{l}\text { Sexual orientation } \\
(n=390)\end{array}$} & Gay (all genders) & 287 & 74 \\
\hline & gbMSM & 229 & 59 \\
\hline & Straight & 103 & 26 \\
\hline \multirow{3}{*}{ Income $(n=348)$} & Less than $\$ 20 \mathrm{~K}$ per year & 60 & 17 \\
\hline & $\$ 20 \mathrm{~K}-\$ 75 \mathrm{~K}$ per year & 176 & 51 \\
\hline & $\$ 75 K+$ per year & 112 & 32 \\
\hline
\end{tabular}

Table 2: Characteristics of eligible participants for Getakit.ca program (continued)

\begin{tabular}{|c|c|c|c|}
\hline Characteristic & Description & $\mathbf{n}$ & $\%$ \\
\hline \multirow{3}{*}{ Education $(n=391)$} & High school of less & 89 & 23 \\
\hline & $\begin{array}{l}\text { College or bachelor's } \\
\text { degree }\end{array}$ & 219 & 56 \\
\hline & $\begin{array}{l}\text { Advanced university } \\
\text { degree }\end{array}$ & 83 & 21 \\
\hline \multirow{2}{*}{$\begin{array}{l}\text { Has a primary care } \\
\text { provider }(n=392)\end{array}$} & Yes & 264 & 67 \\
\hline & No & 128 & 33 \\
\hline \multirow{2}{*}{$\begin{array}{l}\text { Has completed prior } \\
\text { HIV testing }(n=398)\end{array}$} & Yes & 290 & 73 \\
\hline & No & 108 & 27 \\
\hline \multirow{4}{*}{$\begin{array}{l}\text { Location of prior HIV } \\
\text { testing }(n=281)\end{array}$} & General practitioner's office & 98 & 35 \\
\hline & Public health clinic & 154 & 55 \\
\hline & $\begin{array}{l}\text { Emergency Department or } \\
\text { other hospital setting }\end{array}$ & 6 & 2 \\
\hline & Other & 29 & 10 \\
\hline \multirow{3}{*}{$\begin{array}{l}\text { Number of sexual } \\
\text { partners }(n=382)\end{array}$} & 0 or 1 & 191 & 50 \\
\hline & $2-5$ & 165 & 43 \\
\hline & 6 or more & 26 & 7 \\
\hline \multirow{3}{*}{$\begin{array}{l}\text { Partners' HIV status } \\
(n=389)\end{array}$} & $\begin{array}{l}\text { HIV negative (or no } \\
\text { partners) }\end{array}$ & 280 & 72 \\
\hline & HIV positive & 16 & 4 \\
\hline & Unknown & 93 & 24 \\
\hline \multirow{2}{*}{$\begin{array}{l}\text { Has a history of } \\
\text { substance use }(n=364)\end{array}$} & Yes & 194 & 53 \\
\hline & No & 170 & 47 \\
\hline
\end{tabular}

One hundred fifteen participants identified as women, with $24 \%(n=28)$ belonging to a priority group, which increased to $39 \%(n=45)$ when including women of any racial minority. When comparing participants who identified as men or women, there were significant (bivariate) associations between gender and eight characteristics. Women and men differed based on the following: 1) whether they were members of a priority group $(p<0.001)$; 2$)$ whether they identified as straight or gay $(p<0.001)$; 3 ) whether they had a primary care provider $(p=0.005)$; 4$)$ whether they had prior HIV testing $(p<0.001) ; 5)$ whether they were tested in a public health clinic $(p<0.001)$; 6$)$ whether they reported substance use $(p=0.002) ; 7)$ whether they had more than one sexual partner $(p<0.001)$; or 8$)$ their age $(p=0.029)$. When all significant characteristics were entered into a binary logistic regression model, only priority group status and number of sexual partners remained significant, with women being less likely to belong to racialized groups, use injection drugs and/or be a sexual minority $(\mathrm{OR}=0.04 ; 95 \% \mathrm{Cl}=0.02-0.08)$. Women were also more likely to report having fewer sexual partners than men (OR=0.47; 95\% Cl=0.25-0.92) (Table 3).

For HIV testing history, among all participants, 23.9\% ( $n=95)$ reported no prior testing and an additional $3.3 \%(n=13 / 398)$ were uncertain if they had ever previously undergone HIV 
Table 3: Characteristics of eligible participants who had previously been tested for HIV

\begin{tabular}{|c|c|c|c|c|c|c|c|c|c|}
\hline \multirow{3}{*}{ Characteristics } & \multicolumn{2}{|c|}{ Men } & \multicolumn{2}{|c|}{ Women } & \multicolumn{2}{|c|}{ Bivariate } & \multicolumn{3}{|c|}{ Multivariable ${ }^{a}$ [ref = first] } \\
\hline & \multirow{2}{*}{$\mathbf{n}$} & \multirow{2}{*}{$\%$} & \multirow{2}{*}{$\mathbf{n}$} & \multirow{2}{*}{$\%$} & \multirow{2}{*}{$X^{2}$} & \multirow{2}{*}{$p$} & \multirow{2}{*}{ OR } & \multicolumn{2}{|c|}{$95 \% \mathrm{Cl}$} \\
\hline & & & & & & & & Lower & Upper \\
\hline \multicolumn{10}{|l|}{ Priority population } \\
\hline Member of a priority population & 244 & 90 & 28 & 24 & 167.404 & $<0.001$ & 0.04 & 0.02 & 0.08 \\
\hline \multicolumn{10}{|l|}{ Ethnicity } \\
\hline White & 177 & 65 & 79 & 69 & \multirow{3}{*}{3.977} & \multirow{3}{*}{ NS } & \multirow{3}{*}{$\mathrm{N} / \mathrm{A}$} & \multirow{3}{*}{$\mathrm{N} / \mathrm{A}$} & \multirow{3}{*}{ N/A } \\
\hline Black or Indigenous & 23 & 9 & 15 & 13 & & & & & \\
\hline Other & 71 & 26 & 21 & 18 & & & & & \\
\hline \multicolumn{10}{|l|}{ Sexual orientation } \\
\hline Gay & 229 & 85 & 53 & 47 & \multirow{2}{*}{58.970} & \multirow{2}{*}{$<0.001$} & $\mathrm{NC}$ & $N / A$ & $N / A$ \\
\hline Straight & 41 & 15 & 60 & 53 & & & NSS & IN/A & IN/A \\
\hline Income & & & & & & & & & \\
\hline Less than $\$ 20 \mathrm{~K}$ per year & 38 & 16 & 20 & 20 & & & & & \\
\hline$\$ 20 \mathrm{~K}$ to $<\$ 75 \mathrm{~K}$ per year & 120 & 51 & 48 & 48 & 0.757 & NS & N/A & $\mathrm{N} / \mathrm{A}$ & N/A \\
\hline$\$ 75 \mathrm{~K}+$ per year & 78 & 33 & 32 & 32 & & & & & \\
\hline Education & & & & & & & & & \\
\hline High school & 54 & 20 & 34 & 30 & & & & & \\
\hline College or university & 153 & 58 & 57 & 50 & 4.262 & NS & N/A & N/A & $\mathrm{N} / \mathrm{A}$ \\
\hline Advanced degree & 59 & 22 & 22 & 20 & & & & & \\
\hline Healthcare provider & & & & & & & & & \\
\hline Has a primary healthcare provider & 168 & 63 & 88 & 78 & 7.837 & 0.005 & NS & $\mathrm{N} / \mathrm{A}$ & N/A \\
\hline HIV testing & & & & & & & & & \\
\hline Has history of prior HIV testing & 216 & 80 & 63 & 55 & 25.705 & $<0.001$ & NS & $\mathrm{N} / \mathrm{A}$ & $\mathrm{N} / \mathrm{A}$ \\
\hline Location of testing & & & & & & & & & \\
\hline Public health clinic & 131 & 63 & 16 & 26 & & & & & \\
\hline General Practitioner's office & 57 & 27 & 38 & 62 & 28.076 & $<0.001$ & $N / A^{b}$ & N/A & $\mathrm{N} / \mathrm{A}$ \\
\hline Other & 21 & 10 & 7 & 12 & & & & & \\
\hline Number of sexual partners & & & & & & & & & \\
\hline 0 or 1 & 102 & 39 & 80 & 72 & & & & & \\
\hline 2 to 5 & 133 & 51 & 29 & 26 & 34.334 & $<0.001$ & 0.47 & 0.25 & 0.88 \\
\hline 6 or more & 24 & 7 & 2 & $<1$ & & & & & \\
\hline Partner HIV status & & & & & & & & & \\
\hline Negative or no partner & 187 & 71 & 84 & 75 & & & & & \\
\hline Positive & 15 & 6 & 1 & $<1$ & 4.435 & NS & $\mathrm{N} / \mathrm{A}$ & $\mathrm{N} / \mathrm{A}$ & N/A \\
\hline Unknown & 63 & 24 & 27 & 24 & & & & & \\
\hline Substance use & & & & & & & & & \\
\hline Reported substance use & 146 & 59 & 44 & 42 & 9.244 & 0.002 & NS & N/A & $\mathrm{N} / \mathrm{A}$ \\
\hline Age & & & & & & & & & \\
\hline Younger than 25 years & 63 & 24 & 42 & 37 & & & & & \\
\hline 26 to 49 years & 182 & 68 & 67 & 58 & 7.097 & 0.029 & NS & N/A & $\mathrm{N} / \mathrm{A}$ \\
\hline $50+$ years & 22 & 8 & 6 & 2 & & & & & \\
\hline
\end{tabular}


testing. Among the 290 participants who reported prior HIV testing, 59.6\% $(n=174)$ did so fewer than 12 months ago. For testing site ( $n=281$ reported), $54.8 \%(n=154)$ indicated they were last tested in a public health or sexually transmitted infection clinic, 33.6\% $(n=98)$ tested with a primary care provider and $2.1 \%(n=6)$ tested in an emergency department or other hospital setting (Table 2).

Participants who have previously been tested for HIV were more likely to be older $(p<0.005)$, identify as men $(p<0.005)$, have 2-5 sexual partners $(p<0.005)$ and know their sexual partners' HIV-status (Table 4). While $46 \%(n=50)$ of first-time testers were members of a priority population, $82 \%$ of all members of priority populations reported having previously completed HIV testing. Further, participants who were not members of a priority population were nearly five times more likely to be tested for
HIV at a primary care provider clinic compared to a public health clinic ( $p<0.001 ;$ OR $4.71 ; 95 \% \mathrm{Cl}=2.39-9.27$ ). These results identified differences in healthcare access for women versus men.

Overall, $57.1 \%(n=228)$ of participants reported their HIV self-test results back through GetaKit.ca, with $77.6 \%(n=177)$ being negative, $20.6 \%(n=47)$ being invalid, 1.3\% $(n=3)$ being "prefer not to report" and $0.4 \%(n=1)$ being positive. The positivity rate was $0.24 \%$ for all tests $(n=1)$ and $0.44 \%$ for reported results $(n=1)$. There were no significant relationships between participant characteristics and HIV test results. Participants who identified as straight were less likely to report their HIV test result compared to participants who identified as gbMSM $(p<0.05$; OR .58; 95\% Cl=0.37-0.91).

Table 4: Characteristics of eligible GetaKit participants who have previously been tested for HIV

\begin{tabular}{|c|c|c|c|c|c|c|c|c|c|}
\hline \multirow{3}{*}{ Characteristic } & \multirow{3}{*}{ Interpretation } & \multicolumn{4}{|c|}{ Bivariate } & \multicolumn{4}{|c|}{ Multivariable ${ }^{a}$ [ref = first] } \\
\hline & & \multirow{2}{*}{$p$} & \multirow{2}{*}{ OR } & \multicolumn{2}{|c|}{$95 \% \mathrm{Cl}$} & \multirow{2}{*}{$p$} & \multirow{2}{*}{ OR } & \multicolumn{2}{|c|}{$95 \% \mathrm{Cl}$} \\
\hline & & & & Lower & Upper & & & Lower & Upper \\
\hline $\begin{array}{l}\text { Priority } \\
\text { population }\end{array}$ & $\begin{array}{l}\text { Members of priority populations } \\
\text { more likely }\end{array}$ & $<0.05$ & 4.74 & 2.95 & 6.64 & NS & $\mathrm{N} / \mathrm{A}$ & $N / A$ & N/A \\
\hline \multirow{2}{*}{ Age } & 26-49 years old & \multirow{2}{*}{$<0.05$} & 4.85 & 2.96 & 7.93 & \multirow{2}{*}{$<0.005$} & 4.58 & 2.63 & 8.00 \\
\hline & $50+$ years old more likely & & 4.86 & 1.72 & 13.71 & & 9.13 & 2.64 & 31.49 \\
\hline Race & No difference & NS & NS & NS & NS & NS & NS & NS & NS \\
\hline Gender & Women less likely & $<0.05$ & 0.30 & 0.19 & 0.49 & NS & N/A & N/A & $\mathrm{N} / \mathrm{A}$ \\
\hline $\begin{array}{l}\text { Sexual } \\
\text { orientation }\end{array}$ & $\begin{array}{l}\text { Persons who identify as straight } \\
\text { less likely }\end{array}$ & $<0.05$ & 0.25 & 0.15 & 0.40 & $<0.005$ & 0.33 & 0.18 & 0.58 \\
\hline Income & $\begin{array}{l}\text { Persons with a yearly income } \\
\text { between } \$ 20 \mathrm{~K} \text { and } \$ 75 \mathrm{~K} \text { more } \\
\text { likely }\end{array}$ & $<0.05$ & 2.07 & 1.07 & 3.97 & NS & N/A & $\mathrm{N} / \mathrm{A}$ & N/A \\
\hline \multirow{2}{*}{ Education } & $\begin{array}{l}\text { Persons with college/university } \\
\text { education }\end{array}$ & \multirow{2}{*}{$<0.05$} & 2.62 & 1.56 & 4.43 & \multirow{2}{*}{ NS } & \multirow{2}{*}{$N / A$} & \multirow{2}{*}{$\mathrm{N} / \mathrm{A}$} & \multirow{2}{*}{$\mathrm{N} / \mathrm{A}$} \\
\hline & $\begin{array}{l}\text { Persons with advanced degrees } \\
\text { more likely }\end{array}$ & & 3.92 & 1.93 & 8.00 & & & & \\
\hline Primary care & No difference & NS & NS & NS & NS & NS & NS & NS & NS \\
\hline \multirow{2}{*}{$\begin{array}{l}\text { Number of } \\
\text { partners }\end{array}$} & $2-5$ & \multirow{2}{*}{$<0.05$} & 3.16 & 1.91 & 5.24 & $<0.005$ & \multirow{2}{*}{2.89} & \multirow{2}{*}{1.57} & \multirow{2}{*}{5.32} \\
\hline & $6+$ more likely & & 3.4 & 1.13 & 10.27 & NS & & & \\
\hline $\begin{array}{l}\text { Partner HIV } \\
\text { status }\end{array}$ & $\begin{array}{l}\text { Persons who do not know } \\
\text { partners HIV status are less likely }\end{array}$ & $<0.05$ & 0.39 & 0.24 & 0.65 & $<0.005$ & 0.28 & 0.15 & 0.53 \\
\hline Substance use & $\begin{array}{l}\text { Persons with a history are more } \\
\text { likely }\end{array}$ & $<0.05$ & 2.34 & 1.45 & 3.76 & NS & $N / A$ & N/A & $\mathrm{N} / \mathrm{A}$ \\
\hline Reported result & No difference & NS & NS & NS & NS & NS & NS & NS & NS \\
\hline
\end{tabular}

Abbreviations: N/A, not applicable; NS, non-significant

a Hosmer-Lemeshow test $p=0.387$ 


\section{Discussion}

During Phase 1 of GetaKit, 1,268 persons assessed their eligibility; half were eligible, and one-third ordered a test. The most common ineligibility reason was living outside Ottawa. Nearly three-quarters of eligible participants (about one-quarter of eligible women) belonged to priority groups and nearly half of first-time testers were members of priority groups. Priority group participants were more likely to report results, compared with non-priority participants. About one-quarter of all eligible participants (almost one-half of women participants) reported no prior HIV testing. Over half of the participants reported their HIV self-test result back through the GetaKit website; most results were negative and one was positive, for a positivity rate of $0.24 \%$ ( $0.44 \%$ for reported tests) - compared to a baseline HIV positivity rate of $0.08 \%$ in Ottawa.

Consistent with previously published studies, our results highlight that an online ordering system for free HIV self-tests can facilitate testing for some persons affected by HIV (10-15). Supporting this assertion is that nearly three-quarters of our participants were members of a priority group and that our positivity rates were 3-5.5 times higher than the baseline rate in Ottawa. Notably, data from the local health unit indicated that, during the study period, there were 32 reported HIV diagnosis, of whom 13 had been previously diagnosed in other jurisdictions and were aware of their HIV-positive status. As such, GetaKit accounted for 5.2\% $(n=1)$ of new diagnoses in Ottawa during the Phase 1 study period. This outcome is likely related to the fact that over half of our participants identified as gbMSM, which is the group that accounts for over three-quarters of new HIV infections (defined as having been acquired within the preceding 12 months) in Ottawa (16).

\section{Limitations}

Our findings also highlighted facilitated access to testing for women who had not previously been tested for HIV. Indeed, nearly half of participants who identified as women indicated no prior testing. However, no women tested positive for HIV and only one-quarter of women belonged to priority groupssignalling that more efforts are required to target testing at women most at-risk for and affected by HIV. This would include women who are African, Caribbean or Black, Indigenous, use drugs, are transgender, and have other social/economic factors that increase their vulnerability to HIV. One reason why uptake was lower among women may have been the risk of violence associated with receiving an HIV self-test at-home (17). Another reason for lowered participation in GetaKit may have been that women were accessing testing through traditional healthcare venues. That HIV prevention services are often targeted at gbMSM may have also affected uptake among women. Phase 2 of the GetaKit program includes curbside pick-up and ordering at discrete community locations, which will address inadvertent inaccessibly for women who are high-risk for HIV acquisition.
Another important limitation for this study was that it occurred during the coronavirus disease 2019 pandemic (COVID-19), when access to HIV testing services was limited. As such, people may have used GetaKit at a higher rate than would have occurred had healthcare settings been accessible. Conversely, the requirement for access to testing during the study period may have been lower if persons had restricted their sexual practices due to COVID-19 isolation protocols. As well, our findings about women may have been affected by the stepwise deletion process, as this reduced the analytic sample for women due to missing data. To address this, in Phase 2 we added more questions regarding persons who are transgender and gender non-conforming. Next, the proportion of women who belonged to priority groups may have been higher than we identified, as our Phase 1 survey did not inquire about sex work. This has been corrected for Phase 2. Lastly, that GetaKit operated exclusively through a website likely restricted access to persons with lower tech literacy or those who did not have ready access to computers. While COVID-19 restrictions did not allow in-person registration, paper-based surveys have been produced for Phase 2 and will be available at select on-site locations.

\section{Conclusion}

The GetaKit study was the first free mail-out HIV self-testing study in Canada. During Phase 1, we had good interest and uptake among member of the groups most affected by HIV in Canada and among persons never previously tested for HIV. While achieving such outcomes, GetaKit nevertheless seemed to have primarily reached more educated, higher income gbMSM, rather than the full spectrum of HIV priority groups. Thus, our findings highlight the importance of providing HIV self-testing in this manner, while also identifying the pressing need both to scale-up GetaKit to more regions and to reduce barriers to access (as will be addressed in Phase 2). Our findings also highlight the need to expand access to women who are most at-risk for HIV. This could occur through direct outreach and by having more discrete mechanisms for ordering and pick-up (Phase 2). Through such improvements, we may decrease the proportion of persons unaware they are HIV-positive and may help Canada move toward the United Nations 95-95-95 goals.

\section{Authors' statement}

POB is the principal investigator for GetaKit and was involved in all aspects of the project and article. AM was involved in all aspects of GetaKit and this article. AV was involved in all statistical analyses; she wrote all statistical text and reviewed and approved the final manuscript. $\mathrm{NH}$ was a research assistant for GetaKit, and was involved in data collection, article writing, editing, submission, and approval. LO was the HIV clinical lead for GetaKit and a research assistant for GetaKit, and was involved in data collection, article writing, editing, submission, and approval. $\mathrm{MH}$ was a research assistant for GetaKit, and was 
involved in data collection, article writing, editing, submission, and approval. VP was a clinician involved in GetaKit; she also oversaw all social media during phase 1 of GetaKit; VP was involved in data collection, article writing, editing, submission, and approval.

The content and view expressed in this article are those of the authors and do not necessarily reflect those of the Government of Canada.

\section{Competing interests}

None.

\section{Acknowledgements}

POB would like to thank the Ontario HIV Treatment Network for his research chair in public health and HIV prevention. LO would like to thank the Canadian Institutes of Health Research for her Vanier Scholarship.

\section{Funding}

The Ontario HIV Treatment Network funded this project.

\section{References}

1. Joint United Nations Programme on HIV/AIDS. Fast-track: Ending the AIDs epidemic by 2030. Geneva (Switzerland): UNAIDS; 2014 (accessed 2021-05-03). https://www.unaids.org/en/resources/ documents/2014/JC2686_WAD2014report

2. Public Health Agency of Canada. Estimates of HIV incidence, prevalence and Canada's progress on meeting the 90-90-90 HIV targets. Ottawa (ON): PHAC; (updated 2021-06; accessed 2021-05-03). https://www.canada.ca/en/public-health/services/ publications/diseases-conditions/summary-estimates-hivincidence-prevalence-canadas-progress-90-90-90.html

3. Ontario HIV Treatment Network. Priority populations. Toronto (ON): OHTN (accessed 2021-05-04). https://www.ohtn.on.ca/ research-portals/priority-populations/

4. Haddad N, Weeks A, Robert A, Totten S. HIV in Canada-surveillance report, 2019. Can Commun Dis Rep 2021;47(1):77-86. DOI PubMed

5. Laprise C, Bolster-Foucault C. Understanding barriers and facilitators to HIV testing in Canada from 2009-2019: A systematic mixed studies review. Can Commun Dis Rep 2021;47(2):105-25. DOI PubMed

6. Bjørnshagen V, Moseng BU, Ugreninov E. Who do you reach? A Norwegian pilot project on HIV self-testing that targeted men who have sex with men. AIDS Behav 2020;24(2):568-79. DOI PubMed
7. Johnson CC, Kennedy C, Fonner V, Siegfried N, Figueroa C, Dalal S, Sands A, Baggaley R. Examining the effects of HIV self-testing compared to standard HIV testing services: a systematic review and meta-analysis. J Int AIDS Soc 2017;20(1):21594. DOI PubMed

8. Witzel TC, Eshun-Wilson I, Jamil MS, Tilouche N, Figueroa C, Johnson CC, Reid D, Baggaley R, Siegfried N, Burns FM, Rodger AJ, Weatherburn P. Comparing the effects of HIV self-testing to standard HIV testing for key populations: a systematic review and meta-analysis. BMC Med 2020;18(1):381. DOI PubMed

9. Witzel TC, Wright T, McCabe L, Gabriel MM, Wolton A, Gafos M, Ward D, Lampe FC, Phillips AN, Trevelion R, Collaco-Moraes Y, Harbottle J, Speakman A, Bonell C, Dunn DD, McCormack S, Burns FM, Weatherburn P, Rodger AJ. Impact and acceptability of HIV self-testing for trans men and trans women: A mixed-methods subgroup analysis of the SELPHI randomised controlled trial and process evaluation in England and Wales. EClinicalMedicine 2021;32:100700. DOI PubMed

10. Bell SF, Dean JA, Lemoire J, Debattista J, Driver G, Gilks CF, Redmond A, Williams OD. Integrated HIV self-testing (HIVST) service delivery in Queensland for policy and service development: study protocol. AIDS Care 2019;31(2):207-15. DOI PubMed

11. De Boni RB, Veloso VG, Fernandes NM, Lessa F, Corrêa RG, Lima RS, Cruz M, Oliveira J, Nogueira SM, de Jesus B, Reis T, Lentini N, Miranda RL, Bingham T, Johnson CC, Barbosa Junior A, Grinsztejn B. An internet-based HIV self-testing program to increase HIV testing uptake among men who have sex with men in Brazil: descriptive cross-sectional analysis. J Med Internet Res 2019;21(8):e14145. DOI PubMed

12. Dean J, Lui C, Mutch A, Scott M, Howard C, Lemoire J, Crothers A, Fitzgerald L, Williams OD. Knowledge and awareness of HIV self-testing among Australian gay and bisexual men: a comparison of never, sub-optimal and optimal testers willingness to use. AIDS Care 2019;31(2):224-9. DOI PubMed

13. Giacomazzo A. HIV self-testing: Learning from international program models to increase testing. Toronto (ON): CATIE; 2020 (accessed 2021-05-04). https://www.catie.ca/en/pif/fall-2020/ hiv-self-testing-learning-international-program-models-increasetesting

14. Han L, Bien $\mathrm{CH}$, Wei C, Muessig KE, Yang M, Liu F, Yang L, Meng G, Emch ME, Tucker JD. HIV self-testing among online MSM in China: implications for expanding HIV testing among key populations. J Acquir Immune Defic Syndr 2014;67(2):216-21. DOI PubMed

15. LeGrand $\mathrm{S}$, Muessig KE, Horvath KJ, Rosengren AL, Hightow-Weidman LB. Using technology to support HIV self-testing among MSM. Curr Opin HIV AIDS 2017;12(5):425-31. DOI PubMed

16. Spatz Friedman D, O'Byrne P, Roy M. Comparing those diagnosed early versus late in their HIV infection: implications for public health. Int J STD AIDS 2017;28(7):693-701. DOI PubMed

17. World Health Organization. Guidelines on HIV self-testing and partner notification. Geneva, Switzerland: WHO; 2016 (accessed 2021-05-04). https://apps.who.int/iris/bitstream/hand le/10665/251655/9789241549868-eng.pdf 